\title{
Vector Rayleigh Diffraction of High-Power Laser Diode Beam in Optical Communication
}

\author{
Qiang Xu $\mathbb{D}^{D}$, Renxian Li ${ }^{\mathbb{D}}$, Yuanyuan Zhang ${ }^{\mathbb{D}}$, Yiping Han, and Zhensen Wu \\ School of Physics and Optoelectronic Engineering, Xidian University, Xi'an 710071, China \\ Correspondence should be addressed to Qiang Xu; qxu@xidian.edu.cn
}

Received 19 April 2020; Revised 6 June 2020; Accepted 22 June 2020; Published 18 July 2020

Guest Editor: Chen Chen

Copyright ( 92020 Qiang Xu et al. This is an open access article distributed under the Creative Commons Attribution License, which permits unrestricted use, distribution, and reproduction in any medium, provided the original work is properly cited.

Laser diodes (LDs) are widely used in optical wireless communication (OWC) and optical networks, and proper theoretical models are needed to precisely describe the complicated beam field of LDs. A novel mathematical model is proposed to describe the vectorial field of nonparaxial LD beams. Laser beam propagation is studied using the vector Rayleigh diffraction integrals, and the stationary phase method is used to find the asymptotic expansion of diffraction integral. The far-field distribution of the LD beam in the plane parallel and perpendicular to the junction is considered in detail, and the computed intensity distributions of the theory are compared with the corresponding measurements. This model is precise for single transverse model beam of LDs and can be applied to describe the LD beams in OWC and optical networks.

\section{Introduction}

Considering that laser diodes (LDs) are the efficient light source and easy to integrate, LD-enabled optical wireless communication (OWC) is an emerging technology for realizing highconfidentiality and high-speed point-to-point (PtP), vehicle-tovehicle, and white-lighting data access links in free-space communication [1-6], indoor communication [7, 8], underwater communication [9-11], and optical networks [12-16].

However, the output beam quality of LDs is relatively poor, such as astigmatism, high beam asymmetry, and large beam divergence [17-19], in many applications, and proper theoretical models are needed to precisely describe the optical field distribution of LDs.

The problem of laser propagation is mainly dealt through paraxial approximation. However, the output facet of LDs is extremely small, and their beams are divergent and asymmetrical. The rigid optical field distributions cannot be calculated from the paraxial approximation, and the longitudinal component in beam propagation direction should be considered. Thus, the vector theory for nonparaxial beams should be used to precisely describe beam fields of LDs. Several models, such as exponential Gaussian function [20-22], Hermite-Gaussian model [23], nonparaxial diffraction of vectorial Gaussian wave $[24,25]$, plane waves with a small aperture [26], propagation of LD beams in the optical system [27-29], and polarization of LD beams [30], are used to describe the beam field of LDs. However, no theoretical model is used for all cases because of the complicated beam field of LDs. Thus, a novel model should be developed to precisely describe the output field of LDs, which is the aim of this paper.

\section{Vectorial Electric Field of LD Beam}

Considering that transverse electric modes are usually excited in LD, $E(x, y)$ is identified with the component $E_{y}(x, y)$ of the electric field vector, and a source beam is linearly polarized at the plane $z=0$ :

$$
\begin{aligned}
& E_{x}\left(x_{0}, y_{0}\right)=0, \\
& E_{y}\left(x_{0}, y_{0}\right)=E_{0} \exp \left(-p\left|x_{0}\right|^{(3 / 2)}-q y_{0}^{2}\right),
\end{aligned}
$$

where $p$ and $q$ are related to the waveguide structure of LDs, $(1 / p)=1.22\left(\lambda_{n} / d_{x}\right) \times 10^{-3}$ and $(1 / q)=1.22\left(\lambda_{n} / d_{y}\right) \times 10^{-3}$, in which $\lambda_{n}$ is the beam wavelength in the active layer of LDs, $d_{x}$ is the waveguide width in the $x$ direction, and $d_{y}$ is the waveguide width in the $y$ direction, and $E_{0}$ is a constant. 
Beam propagation is governed by the vector Rayleigh diffraction integrals that provide the field expression in the entire half-space $z>0$. When the boundary condition at the plane $z=0$ is given, the field takes the following form $[24,26]$ :

$$
\begin{aligned}
& E_{x}(\mathbf{r})=-\frac{1}{2 \pi} \int E_{x}\left(\mathbf{r}_{0}\right) \frac{\partial G\left(\mathbf{r}, \mathbf{r}_{0}\right)}{\partial z} \mathrm{~d} s, \\
& E_{y}(\mathbf{r})=-\frac{1}{2 \pi} \int E_{y}\left(\mathbf{r}_{0}\right) \frac{\partial G\left(\mathbf{r}, \mathbf{r}_{0}\right)}{\partial z} \mathrm{~d} s, \\
& E_{z}(\mathbf{r})=\frac{1}{2 \pi} \int\left[E_{x}\left(\mathbf{r}_{0}\right) \frac{\partial G\left(\mathbf{r}, \mathbf{r}_{0}\right)}{\partial x}+E_{y}\left(\mathbf{r}_{0}\right) \frac{\partial G\left(\mathbf{r}, \mathbf{r}_{0}\right)}{\partial y}\right] \mathrm{d} s,
\end{aligned}
$$

where $\mathbf{r}_{0}=x_{0} \mathbf{i}+y_{0} \mathbf{j}$ ( $\mathbf{r}_{0}$ is the vector in beam output plane), $\mathbf{r}=x \mathbf{i}+y \mathbf{j}+z \kappa$ ( $\mathbf{r}$ is the beam propagation vector), $\mathbf{i}, \mathbf{j}, \kappa$ are the unit vectors in the $x$-, $y$-, and $z$-directions, respectively, and

$$
G\left(\mathbf{r}, \mathbf{r}_{0}\right)=\frac{\exp \left(i k\left|\mathbf{r}-\mathbf{r}_{0}\right|\right)}{\left|\mathbf{r}-\mathbf{r}_{0}\right|}
$$

in which $k$ is the wavenumber related to wavelength $\lambda$ by $k=2 \pi / \lambda$. Substituting equation (4) into equation (3) yields $[24,26]$

$$
\begin{aligned}
E_{x}(x, y, z)= & -\frac{1}{2 \pi} \int_{-\infty}^{+\infty} \int_{-\infty}^{+\infty} E_{x}\left(x_{0}, y_{0}\right) z \frac{i k\left|\mathbf{r}-\mathbf{r}_{0}\right|-1}{\left|\mathbf{r}-\mathbf{r}_{0}\right|^{3}} \exp \left(i k\left|\mathbf{r}-\mathbf{r}_{0}\right|\right) \mathrm{d} x_{0} \mathrm{~d} y_{0} \\
E_{y}(x, y, z)= & -\frac{1}{2 \pi} \int_{-\infty}^{+\infty} \int_{-\infty}^{+\infty} E_{y}\left(x_{0}, y_{0}\right) z \frac{i k\left|\mathbf{r}-\mathbf{r}_{0}\right|-1}{\left|\mathbf{r}-\mathbf{r}_{0}\right|^{3}} \exp \left(i k\left|\mathbf{r}-\mathbf{r}_{0}\right|\right) \mathrm{d} x_{0} \mathrm{~d} y_{0} \\
E_{z}(x, y, z)= & \frac{1}{2 \pi} \int_{-\infty}^{+\infty} \int_{-\infty}^{+\infty}\left[E_{x}\left(x_{0}, y_{0}\right)\left(x-x_{0}\right) \frac{i k\left|\mathbf{r}-\mathbf{r}_{0}\right|-1}{\left|\mathbf{r}-\mathbf{r}_{0}\right|^{3}} \exp \left(i k\left|\mathbf{r}-\mathbf{r}_{0}\right|\right)\right. \\
& \left.+E_{y}\left(x_{0}, y_{0}\right)\left(y-y_{0}\right) \frac{i k\left|\mathbf{r}-\mathbf{r}_{0}\right|-1}{\left|\mathbf{r}-\mathbf{r}_{0}\right|^{3}} \exp \left(i k\left|\mathbf{r}-\mathbf{r}_{0}\right|\right)\right] \mathrm{d} x_{0} \mathrm{~d} y_{0} .
\end{aligned}
$$

We expand $\exp \left(i k\left|\mathbf{r}-\mathbf{r}_{0}\right|\right)$ into a series, keeping the first, second-, and third-order series expansions [31]:

$$
\exp \left(i k\left|\mathbf{r}-\mathbf{r}_{0}\right|\right) \approx \exp \left[i k r-i k \frac{x x_{0}+y y_{0}}{r}+i k \frac{\left(y^{2}+z^{2}\right) x_{0}^{2}+\left(x^{2}+z^{2}\right) y_{0}^{2}-2 x y x_{0} y_{0}}{2 r^{3}}\right]
$$

where $r=\sqrt{x^{2}+y^{2}+z^{2}}$, replace $\exp \left(i k\left|\mathbf{r}-\mathbf{r}_{0}\right|\right)$ in equation (5) by equation (6), and replace $\left|\mathbf{r}-\mathbf{r}_{0}\right|$ in equation (5) by $r$ :

$$
\begin{aligned}
E_{x}(x, y, z)= & 0, \\
E_{y}(x, y, z)= & -\frac{1}{2 \pi} z \frac{i k r-1}{r^{3}} \exp (i k r) \int_{-\infty}^{+\infty} \int_{-\infty}^{+\infty} E_{y}\left(x_{0}, y_{0}\right) \\
& \times \exp \left[-i k \frac{x x_{0}+y y_{0}}{r}+i k \frac{\left(y^{2}+z^{2}\right) x_{0}^{2}+\left(x^{2}+z^{2}\right) y_{0}^{2}-2 x y x_{0} y_{0}}{2 r^{3}}\right] \mathrm{d} x_{0} \mathrm{~d} y_{0}, \\
E_{z}(x, y, z)= & \frac{1}{2 \pi} \frac{i k r-1}{r^{3}} \exp (i k r) \int_{-\infty}^{+\infty} \int_{-\infty}^{+\infty}\left[E_{x}\left(x_{0}, y_{0}\right)\left(x-x_{0}\right)+E_{y}\left(x_{0}, y_{0}\right)\left(y-y_{0}\right)\right] \\
& \times \exp \left[-i k \frac{x x_{0}+y y_{0}}{r}+i k \frac{\left(y^{2}+z^{2}\right) x_{0}^{2}+\left(x^{2}+z^{2}\right) y_{0}^{2}-2 x y x_{0} y_{0}}{2 r^{3}}\right] \mathrm{d} x_{0} \mathrm{~d} y_{0} .
\end{aligned}
$$

For large $k\left(10^{4} \mathrm{~mm}^{-1}\right), \exp \left[-i k\left(\left(x x_{0}+y y_{0}\right) / r\right)+i k\left(\left(\left(y^{2}\right.\right.\right.\right.$ $\left.\left.\left.\left.+x^{2}\right) x_{0}^{2}+\left(x^{2}+z^{2}\right) y_{0}^{2}-2 x y x_{0} y_{0}\right) / 2 r^{3}\right)\right]$ rapidly oscillates, and such rapid oscillations over the range of integration indicate that the integrand averages to approximately zero, except near the stationary phase. Thus, the stationary phase method is used to find the asymptotic expansion of the diffraction integral.

The corresponding diffraction integral is approximated by [32] 


$$
U(x, y, z)=\iint_{D} f\left(x_{0}, y_{0}\right) \exp \left[i k g\left(x_{0}, y_{0}\right)\right] \mathrm{d} x_{0} \mathrm{~d} y_{0} \approx \frac{2 \pi \sigma}{k \sqrt{|H|}} f\left(x_{s}, y_{s}\right) \exp \left[i k g\left(x_{s}, y_{s}\right)\right]
$$

$f\left(x_{0}, y_{0}\right)=E_{y}\left(x_{0}, y_{0}\right)$ for $E_{y}(x, y, z)$, and $f\left(x_{0}, y_{0}\right)=$ $E_{x}\left(x_{0}, y_{0}\right)\left(x-x_{0}\right)+E_{y}\left(x_{0}, y_{0}\right)\left(y-y_{0}\right)$ for $E_{z}(x, y$, $z)$.where

$$
\begin{aligned}
& H=\frac{\partial^{2} g}{\partial x_{0}^{2}} \frac{\partial^{2} g}{\partial y_{0}^{2}}-\left(\frac{\partial^{2} g}{\partial x_{0} \partial y_{0}}\right)^{2} \\
& \sigma= \begin{cases}1, & (\text { if } H<0), \\
i, & \left(\text { if } H>0,\left.\frac{\partial^{2} g}{\partial x_{0}^{2}}\right|_{x_{s}, y_{s}}>0\right), \\
-i, & \left(\text { if } H>0,\left.\frac{\partial^{2} g}{\partial x_{0}^{2}}\right|_{x_{s}, y_{s}}<0\right),\end{cases}
\end{aligned}
$$

where $x_{s}$ and $y_{s}$ are the stationary phase points, and we have $g\left(x_{0}, y_{0}\right)=-\frac{x x_{0}+y y_{0}}{r}+\frac{\left(y^{2}+z^{2}\right) x_{0}^{2}+\left(x^{2}+z^{2}\right) y_{0}^{2}-2 x y x_{0} y_{0}}{2 r^{3}}$.

Letting

$$
\begin{gathered}
\frac{\partial g}{\partial x_{0}}=0 \\
\frac{\partial g}{\partial y_{0}}=0
\end{gathered}
$$

we find the stationary phase points

$$
\begin{aligned}
x_{s} & =\frac{r^{2}}{z^{2}} x, \\
y_{s} & =\frac{r^{2}}{z^{2}} y, \\
g\left(x_{s}, y_{s}\right) & =\frac{-r}{2 z^{2}}\left(x^{2}+y^{2}\right),
\end{aligned}
$$

and $\left(\partial^{2} g / \partial x_{0}^{2}\right)=\left(\left(y^{2}+z^{2}\right) / r^{3}\right),\left(\partial^{2} g / \partial y_{0}^{2}\right)=\left(\left(x^{2}+z^{2}\right) / r^{3}\right)$, and $\left(\partial^{2} g / \partial x_{0} \partial y_{0}\right)=\left(x y / r^{3}\right)$.

Thus,

$$
H=\frac{\partial^{2} g}{\partial x_{0}^{2}} \frac{\partial^{2} g}{\partial y_{0}^{2}}-\left(\frac{\partial^{2} g}{\partial x_{0} \partial y_{0}}\right)^{2}=\frac{z^{2}}{r^{4}}
$$

and $H>0,\left.\left(\partial^{2} g / \partial x_{0}^{2}\right)\right|_{x_{s}, y_{s}}>0$,

$$
\sigma=i
$$

Substituting equations (13)-(16) into equation (7) yields

$$
\begin{aligned}
& E_{x}(x, y, z)=0 \\
& E_{y}(x, y, z)=-i \frac{i k r-1}{k r} E_{0} \cdot \exp \left(-p \frac{r^{2}}{z^{2}}|x|^{(3 / 2)}-q\left(\frac{r^{2}}{z^{2}} y\right)^{2}\right) \times \exp \left[i k \frac{-r}{2 z^{2}} \frac{x^{2}+y^{2}}{z^{2}}\right] \exp (i k r) \\
& E_{z}(x, y, z)=i \frac{i k r-1}{k r} \cdot \frac{y}{z}\left(\frac{x^{2}+y^{2}}{z^{2}}\right) E_{0} \cdot \exp \left(-p \frac{r^{2}}{z^{2}}|x|^{(3 / 2)}-q\left(\frac{r^{2}}{z^{2}} y\right)^{2}\right) \times \exp \left[i k \frac{-r}{2 z^{2}}\left(x^{2}+y^{2}\right)\right] \exp (i k r)
\end{aligned}
$$

Equation (17) represents the expression of vector theory for nonparaxial $\mathrm{LD}$ beam.

The intensity profiles can be given by

$$
\begin{aligned}
& I_{x}(x, y, z)=0 \\
& I_{y}(x, y, z)=\frac{1+k^{2} r^{2}}{k^{2} r^{2}} E_{0}^{2} \cdot \exp \left(-2 p \frac{r^{2}}{z^{2}}|x|^{(3 / 2)}-2 q\left(\frac{r^{2}}{z^{2}} y\right)^{2}\right), \\
& I_{z}(x, y, z)=\frac{1+k^{2} r^{2}}{k^{2} r^{2}} \frac{y^{2}}{z^{2}}\left(\frac{x^{2}+y^{2}}{z^{2}}\right)^{2} E_{0}^{2} \cdot \exp \left(-2 p \frac{r^{2}}{z^{2}}|x|^{(3 / 2)}-2 q\left(\frac{r^{2}}{z^{2}} y\right)^{2}\right),
\end{aligned}
$$




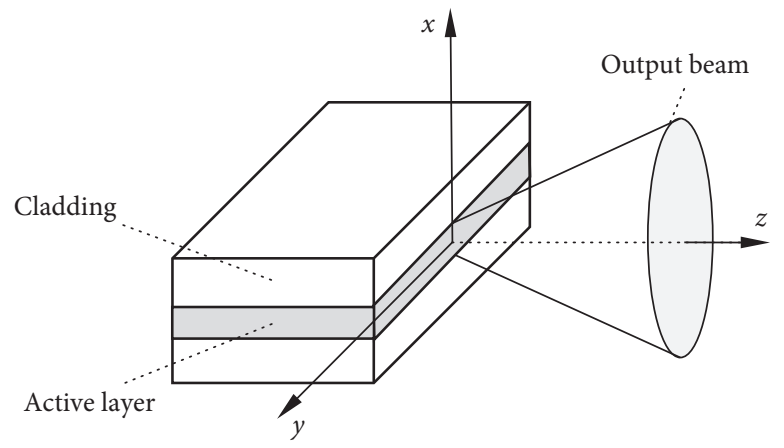

Figure 1: Facet of a LD chip and the related coordinate system.

Table 1: Parameter of LDs.

\begin{tabular}{|c|c|c|c|c|c|}
\hline LDs & Wave model & Operating current (mA) & Wavelength (nm) & Parameter $p$ & Parameter $q$ \\
\hline USHIO HL63391DC & Single transverse model & 225 & 639 & $1.55 \times 10^{3}$ & $2.20 \times 10^{4}$ \\
\hline TOSHIBA TOLD9441MC & Single transverse model & 50 & 650 & 335 & $3.05 \times 10^{4}$ \\
\hline USHIO HL63290HD & Multitransverse model & 2.4 & 638 & 256 & $1.55 \times 10^{4}$ \\
\hline
\end{tabular}

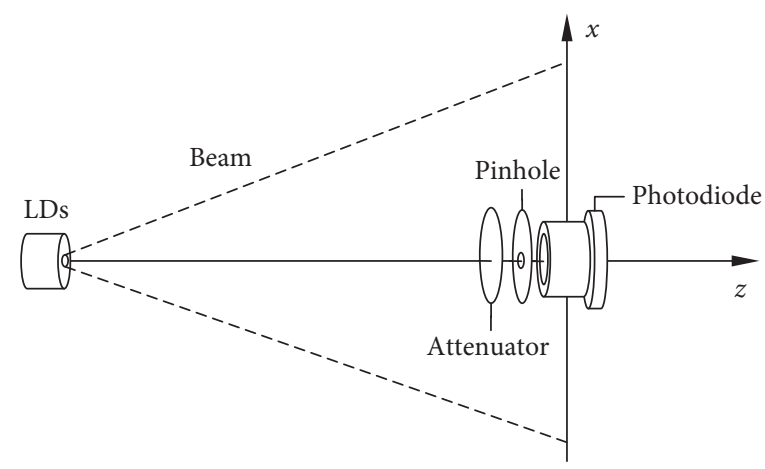

FIgURE 2: Experimental setup.

and the total intensity can be expressed as

$$
\begin{aligned}
I(x, y, z)= & I_{x}(x, y, z)+I_{y}(x, y, z)+I_{z}(x, y, z) \\
= & \frac{1+k^{2} r^{2}}{k^{2} r^{2}}\left[1+\frac{y^{2}}{z^{2}}\left(\frac{x^{2}+y^{2}}{z^{2}}\right)^{2}\right] \\
& \cdot E_{0}^{2} \exp \left(-2 p \frac{r^{2}}{z^{2}}|x|^{(3 / 2)}-2 q\left(\frac{r^{2}}{z^{2}} y\right)^{2}\right) .
\end{aligned}
$$

The intensity of LD beams can be investigated in two vertical planes. In the plane perpendicular to the junction (i.e., $y=0$ ), as shown in Figure 1, the substitution of $y=0$ into equation (19) yields

$$
I(x, 0, z)=\frac{1+k^{2}\left(x^{2}+z^{2}\right)}{k^{2}\left(x^{2}+z^{2}\right)} E_{0}^{2} \exp \left(-2 p \frac{x^{2}+z^{2}}{z^{2}}|x|^{(3 / 2)}\right) .
$$

In the plane parallel to the junction (i.e., $x=0$ ), as shown in Figure 1, the total intensity can be expressed as follows:

$$
I(0, y, z)=\frac{1+k^{2}\left(y^{2}+z^{2}\right)}{k^{2}\left(y^{2}+z^{2}\right)}\left[1+\frac{y^{6}}{z^{6}}\right] E_{0}^{2} \exp \left(-2 q\left(\frac{y^{2}+z^{2}}{z^{2}} y\right)^{2}\right) .
$$

\section{Experimental Procedure}

The experiments were performed to examine the theoretical results using three high-power LDs (USHIO HL63391DC, TOSHIBA TOLD9441MC, and USHIO HL63290HD). The parameters are shown in Table 1.

As shown in Figure 2, the intensity profiles of laser beam were measured through a pinhole scan (radius is $100 \mu \mathrm{m}$ ) and a photodiode (LSGSPD-UL0.25, $0.25 \mathrm{~mm}$ visible light PIN photodiode, wavelength $500-880 \mathrm{~nm}$, and $0.25 \mathrm{~mm}$ active diameter) behind the hole. The photodiode moved along the straight lines parallel to the output facet of the LDs' chip in the $x-z$ and $y-z$ planes, where $z=50 \mathrm{~mm}$. The uncertainty of measurements is less than $1 \%$.

Figure 3 shows the measurements and theoretical beam profiles of HL63391DC, and the intensity curve of the theory agrees with the experimental data in most portions. Figure 4 


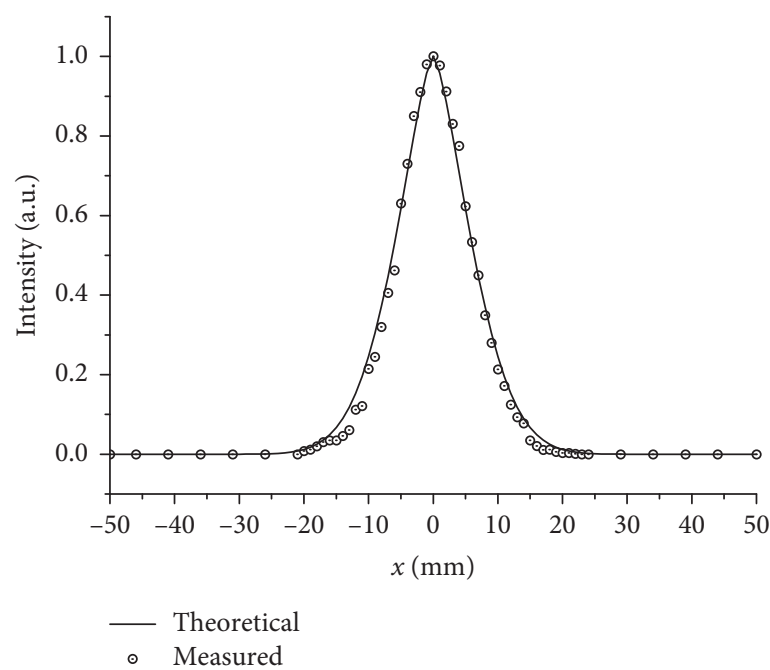

(a)

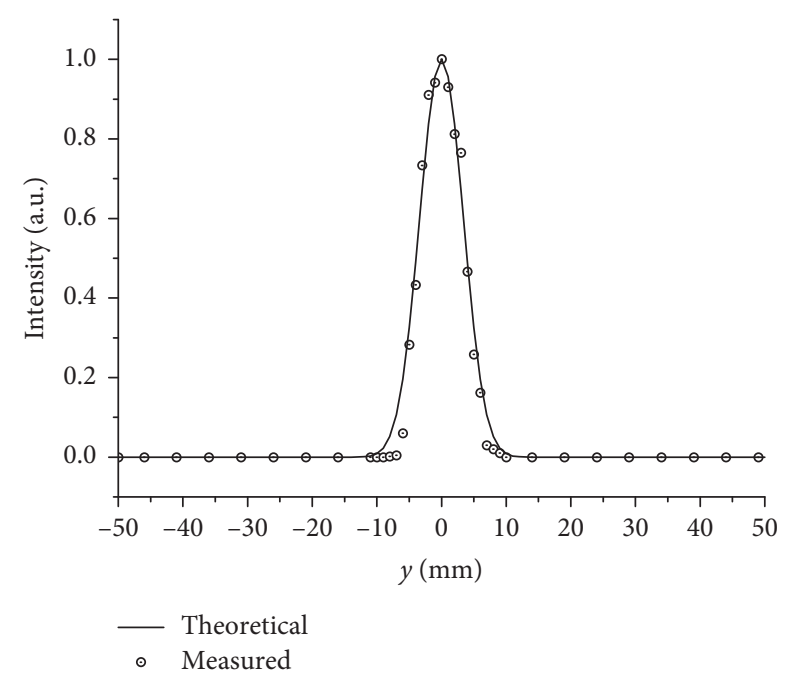

(b)

Figure 3: Measured and theoretical beam intensity profiles of HL63391DC. (a) $x-z$ plane. (b) $y-z$ plane.

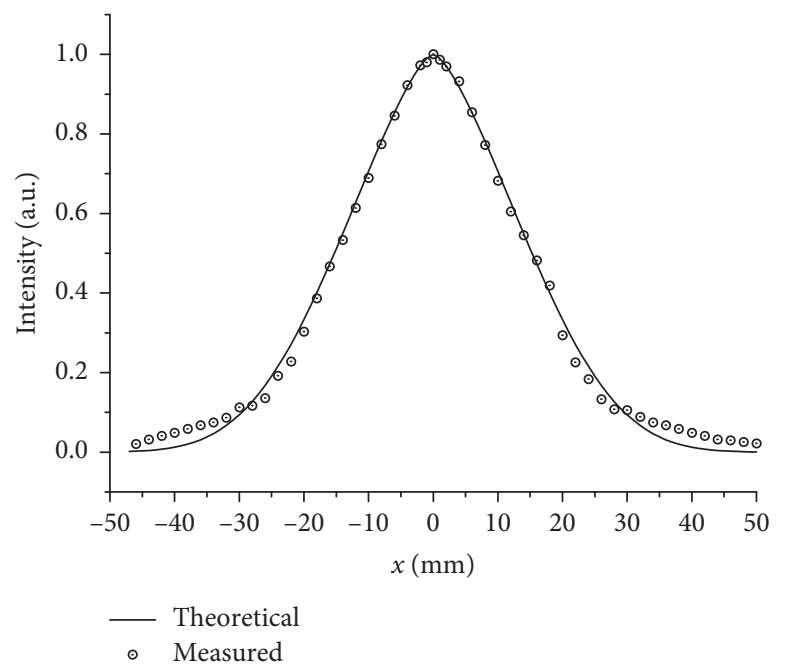

(a)

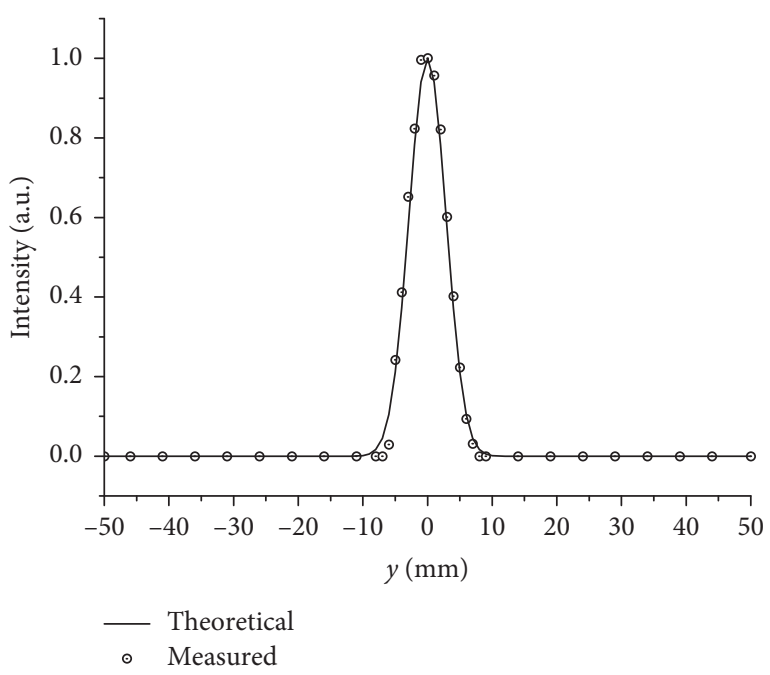

(b)

Figure 4: Measured and theoretical beam intensity profiles of TOLD9441MC. (a) $x-z$ plane. (b) $y-z$ plane.

shows the light intensity profiles of TOLD9441MC. The calculated profiles agree well with the experimental data in most portions, except for the discrepancies in the low-intensity value regions in the $x-z$ plane. The theoretical curve agrees well with the experimental data in the $y-z$ plane. Figure 5 shows the light intensity profiles of HL63290HD, and the discrepancies of theory and measurement of this LD are greater than those of HL63391DC and TOLD9441MC because only single transverse mode exists in HL63391DC and TOLD9441MC, whereas multitransverse modes exist in HL63290HD. Thus, the output light field of the latter is more complicated, the shape intensity of two lobes appears in the $y-z$ plane, and the theoretical curve does not fit the measurement.
Compared with the previous models of LD beam, including Hermite-Gaussian model [23], Gaussian model [25, 27, 28], elliptical Gaussian model [24, 29], and negative exponential Gaussian model $[22,30]$, the novel output model $E_{y}\left(x_{0}, y_{0}\right)=E_{0} \exp \left(-p\left|x_{0}\right|^{(3 / 2)}-q y_{0}^{2}\right)$ in this article is more precise for single transverse model beam. For the calculation of the vector Rayleigh diffraction integrals, we expand $\exp \left(i k\left|\mathbf{r}-\mathbf{r}_{0}\right|\right)$ into a series by keeping the first, second, and third series expansions. The calculations make the diffraction integral of beam distribution with large divergence more reliable compared with the first two expansions in the article $[24,26]$. 


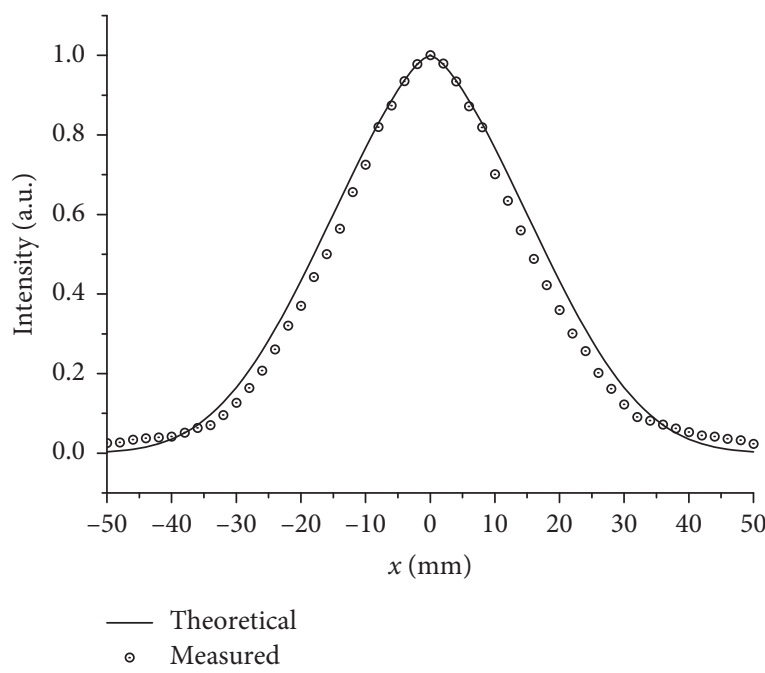

(a)

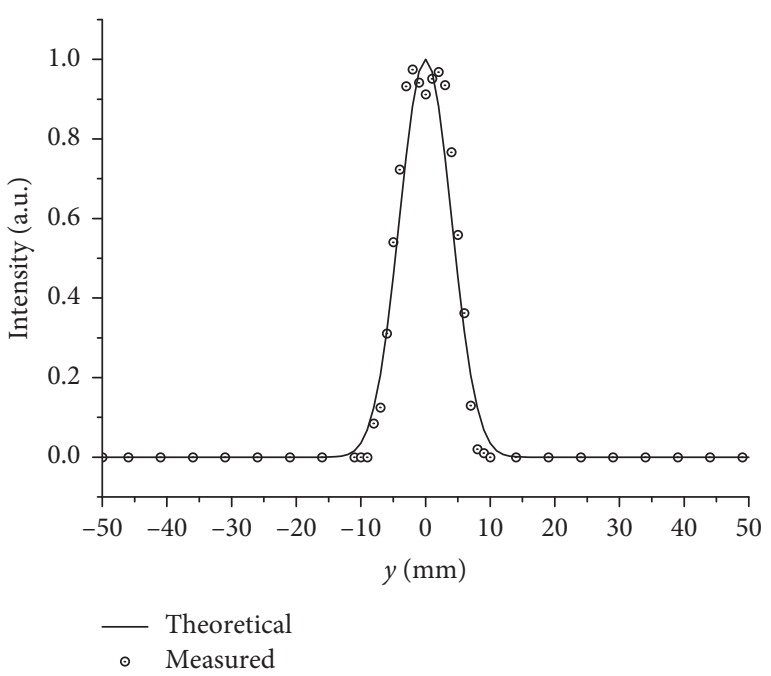

(b)

FIGURE 5: Measured and theoretical beam intensity profiles of HL63290HD. (a) $x-z$ plane. (b) $y-z$ plane.

\section{Conclusion}

A novel theoretical model for the nonparaxial vectorial field of high-power LDs was proposed, and the beam parameters were related to the structure of LDs' waveguide. High-order approximations of the diffraction integral were calculated on the basis of the vector Rayleigh diffraction integrals, the fields parallel and perpendicular to beam propagation direction were considered, and the beam intensities of three high-power LDs beam were measured. The mathematical model provided a good fit to the experimental data of single transverse model beam of LDs. This mathematical model can be used to describe the beam propagation and shape of LDs in OWC and optical networks.

\section{Data Availability}

The data used to support the findings of this study are included within the article.

\section{Conflicts of Interest}

The authors declare that they have no conflicts of interest.

\section{Acknowledgments}

This work was supported by the National Natural Science Foundation of China (61975158) and Aeronautical Science Foundation of China (20180181).

\section{References}

[1] C.-T. Tsai, C.-H. Cheng, H.-C. Kuo, and G.-R. Lin, “Toward high-speed visible laser lighting based optical wireless communications," Progress in Quantum Electronics, vol. 67, Article ID 100225, 2019.

[2] C. Shen, J. A. Holguin-Lerma, A. A. Alatawi et al., "Group-IIInitride superluminescent diodes for solid-state lighting and high-speed visible light communications," IEEE Journal of
Selected Topics in Quantum Electronics, vol. 25, no. 6, pp. 1-10, 2019.

[3] J. Kosman, O. Almer, T. A. Abbas et al., "29.7 A 500 Mb/s$46.1 \mathrm{dBm}$ CMOS SPAD receiver for laser diode visible-light communications," in Proceedings of the IEEE International Solid-State Circuits Conference, pp. 468-470, San Francisco, CA, USA, 2019 February.

[4] M. H. M. Shamim, M. A. Shemis, C. Shen et al., "Analysis of optical injection on red and blue laser diodes for high bit-rate visible light communication," Optics Communications, vol. 449, pp. 79-85, 2019.

[5] S. Gwyn, S. Watson, S. Viola et al., "GaN-based distributed feedback laser diodes for optical communications," in Proceedings of SPIE-The International Society for Optical Engineering, Lisbon, Portugal, 2019.

[6] H.-Y. Wang, Y.-C. Chi, and G.-R. Lin, "Dual-mode laser diode carrier with orthogonal polarization and single-mode modulation for remote-node heterodyne MMW-RoF," Optics Letters, vol. 41, no. 20, pp. 4676-4679, 2016.

[7] C. Li, X. Zhang, E. Tangdiongga et al., "Cost-efficient halfduplex $10 \mathrm{Gbit} / \mathrm{s}$ all-optical indoor optical wireless communication enabled by a low-cost Fabry-Perot laser/photodetector," Optics Letters, vol. 44, no. 5, pp. 1158-1161, 2019.

[8] J. Fakidis, S. Videv, S. Kucera, H. Claussen, and H. Haas, "Indoor optical wireless power transfer to small cells at nighttime," Journal of Lightwave Technology, vol. 34, no. 13, pp. 3236-3258, 2016.

[9] Y. Chen, M. Kong, T. Ali et al., “ $26 \mathrm{~m} / 5.5 \mathrm{Gbps}$ air-water optical wireless communication based on an OFDM-modulated 520-nm laser diode," Optics Express, vol. 25, no. 13, pp. 14760-14765, 2017.

[10] J. Wang, C. Lu, S. Li, and Z. Xu, “ 100 m/500 Mbps underwater optical wireless communication using an NRZ-OOK modulated $520 \mathrm{~nm}$ laser diode," Opt. Express, vol. 27, no. 9, pp. 12171-12181, 2019.

[11] X. Liu, S. Yi, X. Zhou et al., “345 m underwater optical wireless communication with $270 \mathrm{Gbps}$ data rate based on a green laser diode with NRZ-OOK modulation," Optics Express, vol. 25, no. 22, p. 27937, 2017. 
[12] W.-S. Tsai, H.-H. Lu, H.-W. Wu et al., “ 500 Gb/s PAM4 FSOUWOC convergent system with a R/G/B five-wavelength polarization-multiplexing scheme," IEEE Access, vol. 8, pp. 16913-16921, 2020.

[13] A. Majumdar, C. Mandal, and S. Gangopadhyay, "Laser diode to single-mode circular core parabolic index fiber coupling via upside-down tapered hyperbolic microlens on the tip of the fiber: prediction of coupling optics by abcd matrix formalism," Journal of Optical Communications, vol. 40, no. 3, pp. 171-180, 2019.

[14] Fiber optic active components and devices, Performance standards, Part 3, Modulator-integrated laser diode transmitters for $40-G b i t / s$ fiber optic transmission systems, 1-16, 2018.

[15] S. Watson, S. Gwyn, S. Viola et al., "InGaN/GaN laser diodes and their applications," in Proceedings of the 2018 20th International Conference on Transparent Optical Networks, Bucharest, Romania, 2018 July.

[16] N. Kim, M. Park, S. An, T.-S. Kim, W. S. Han, and O.-K. Kwon, "25 Gbps electroabsorption modulated dfb laser diodes for digital fronthaul network," in Proceedings of the 2018 23rd Opto-Electronics and Communications Conference, Jeju Island, Republic of Korea, July 2018

[17] K. Lei, X. Qin, H. Liu, and M. Ni, "Analysis and modeling of melt pool morphology for high power diode laser cladding with a rectangle beam spot," Optics \& Lasers in Engineering, vol. 110, pp. 89-99, 2018.

[18] M. U. Hoque, "Enhancement of optical fiber coupling based on collimated and focused laser diode beam with micro aspherical plano-convex lens fabricated by excimer laser," Fiber \& Integrated Optics, vol. 37, no. 5, pp. 264-276, 2018.

[19] H. Tan, H. Meng, X. Ruan, W. Du, and Z. Wang, "High-power direct diode laser output by spectral beam combining," Laser Physics, vol. 28, no. 3, Article ID 035802, 2018.

[20] X. Zeng and A. Naqwi, "Far-field distribution of doubleheterostructure diode laser beams," Applied Optics, vol. 32, no. 24, pp. 4491-4500, 1993.

[21] S. Nemoto, "Experimental evaluation of a new expression for the far field of a diode laser beam," Applied Optics, vol. 33, no. 27, pp. 6387-6390, 1994.

[22] H. Dong, S. Shi, and S. Chen, "Analysis of error yielded by scalar approximation to the properties of laser diode beams," Applied Optics, vol. 45, no. 21, pp. 5160-5163, 2006.

[23] Z. Zhao, K. Duan, and B. Lü, "Non-equiphase Hermite-Gaussian model of diode laser beams," Optik, vol. 119, no. 4, pp. 167-170, 2008.

[24] K. Duan and B. Lü, "Propagation properties of vectorial elliptical Gaussian beams beyond the paraxial approximation," Optics \& Laser Technology, vol. 36, no. 6, pp. 489-496, 2004.

[25] X. Zeng, C. Liang, and Y. An, "Far-field propagation of an offaxis Gaussian wave," Applied Optics, vol. 38, no. 30, pp. 6253-6256, 1999.

[26] K. Duan and B. Lü, "Nonparaxial diffraction of vectorial plane waves at a small aperture," Optics \& Laser Technology, vol. 37, no. 3, pp. 193-197, 2005.

[27] M. R. H. Rad, F. D. Kashani, M. M. Eftekhari, and M. R. Mahzoun, "Characterizing the divergence properties of the laser diode beams propagation through collimator and aperture ABCD optical system," Optics \& Laser Technology, vol. 42, no. 8, pp. 1269-1275, 2010.

[28] F. Kashani, M. R. H. Rad, Z. Firozzadeh, and M. R. Mahzoun, "Beam propagation analysis of a multilaser diode FSO system through free space," Journal of Optics, vol. 13, no. 10, Article ID 105709, 2011.
[29] H. Sun, "Simple mathematical model for designing laser diode focusing optics with a large numerical aperture," Optical Engineering, vol. 53, no. 10, pp. 105105.1-105105.7, 2014.

[30] Q. Xu, J. Wang, Y. Han, and Z. Wu, "Vectorial analytical description of the polarized light of a high-power laser diode," Applied Optics, vol. 52, no. 8, pp. 1711-1715, 2013.

[31] A. Ciattoni, B. Crosignani, and P. D. Porto, "Vectorial analytical description of propagation of a highly nonparaxial beam," Optics Communications, vol. 202, no. 1-3, pp. 17-20, 2002.

[32] J. Stamnes, "Waves, rays, and the method of stationary phase," Optics Express, vol. 10, no. 16, pp. 740-751, 2002. 